Revue des patrimoines

Le cheval et ses patrimoines (1ère partie)

\title{
Races, types, tribus ou ethnies des chevaux de courses au XIX ${ }^{\mathrm{e}}$ siècle
}

Tanneguy de Sainte Marie

\section{(2) OpenEdition}

Journals

Édition électronique

URL : https://journals.openedition.org/insitu/9683

DOI : 10.4000/insitu.9683

ISSN : 1630-7305

Éditeur

Ministère de la Culture

Référence électronique

Tanneguy de Sainte Marie, "Races, types, tribus ou ethnies des chevaux de courses au XIXe siècle », In Situ [En ligne], 18 | 2012, mis en ligne le 31 juillet 2012, consulté le 21 septembre 2021. URL : http:// journals.openedition.org/insitu/9683; DOI : https://doi.org/10.4000/insitu.9683

Ce document a été généré automatiquement le 21 septembre 2021.

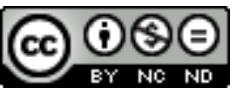

In Situ Revues des patrimoines est mis à disposition selon les termes de la licence Creative Commons Attribution - Pas d'Utilisation Commerciale - Pas de Modification 4.0 International. 


\title{
Races, types, tribus ou ethnies des chevaux de courses au XIX ${ }^{\mathrm{e}}$ siècle
}

\author{
Tanneguy de Sainte Marie
}

1 En France, en ces débuts du XIX ${ }^{e}$ siècle, le cheval n'est, le plus souvent, ni un ami, ni un compagnon comme il l'est en Arabie, ni même une gloire ou une œuvre d'art comme en Angleterre. C'est aux yeux des uns, une chose, un bétail, une pure marchandise, aux yeux des autres un instrument de travail ou un objet de vanité stérile et une occasion de luxe apparent. C'est en France qu'on assiste souvent à ces vicissitudes des destinées chevalines qui débutent par les palmes des champs de courses ou le fracas des batailles et finissent chez l'équarisseur après avoir traversé les degrés décroissants de la chasse, de l'attelage, du fiacre, du cabriolet, de la charrette et enfin, quand ils le peuvent encore, du tourniquet.

2 Ce siècle est celui de toutes les nouveautés, de toutes les inventions. Celui de la lumière, de la photographie, de l'automobile, le siècle de l'avènement de la Belle Époque et des réglementations, donc des débuts d'une certaine standardisation entraînant l'évaporation furtive et progressive des types, très marqués, de chevaux de pays que furent les bidets ambleurs de Corlay, les sommiers, les roussins, les coursiers et autres destriers.

Ce siècle sera également celui de la création des diverses Sociétés d'Encouragement pour l'Amélioration des races de courses (galop plat, obstacle, trot). Simultanément, sous couvert d'amélioration génétique, ce sera le siècle de la disparition progressive d'un certain nombre de phénotypes d'équidés de tous poils.

4 C'est par l'arrêté du 13 fructidor de l'an XIII (31 août 1805) que furent réglementées officiellement les courses hippiques en France. Ainsi sera prescrit leur établissement dans les départements de l'Empire les plus remarquables par la bonté des chevaux qu'on y élève, des prix devant être accordés aux chevaux les plus rapides.

Cette consécration officielle des courses se devait d'effacer l'image d'une époque révolue où ces activités ne jouaient qu'un rôle secondaire. Derniers divertissements de la royauté expirante, les courses hippiques n'étaient qu'un spectacle de gala offert à la noblesse, spectacle où l'initiative privée était seule en cause, sans que d'ailleurs il pût en résulter quelque progrès pour l'amélioration de la race et pour l'élevage national. 
6 À partir de 1806, et conformément aux prescriptions de l'arrêté de fructidor, des courses furent créées dans les départements de l'Orne (Haras du Pin), de la Corrèze (Tulle et Haras de Pompadour), du Morbihan (Haras de Langonnet), de la Seine, de la Sèvre, des Côtes du Nord, des Hautes Pyrénées et autres.

7 Pouvaient prendre part à toutes ces épreuves, les chevaux entiers et juments de 5 à 6 ans révolus, nés en France et montés par des piqueurs français. Aucune notion de race ou de type n'était imposée. Sur les grands hippodromes parisiens seuls les pur-sang étaient concernés alors que sur les champs de courses de province ces réjouissances étaient ouvertes aux bidets locaux.

8 Quatre prix de $2000 \mathrm{~F}$ avaient été attribués annuellement à chacun des départements précités, exception faite pour celui de la Seine qui recevait un prix de $4000 \mathrm{~F}$.

Un certain patriotisme exclusif et un amour-propre national incitaient à s'éloigner autant que possible des théories et des pratiques anglaises. L'emploi du pur-sang anglais comme reproducteur était écarté d'une manière absolue. Ainsi, les courses ne pouvaient que favoriser les moins mauvais représentants de races locales abâtardies, au lieu d'aider à leur amélioration et régénération. On ne tarda pas à s'apercevoir de l'erreur commise et dès 1810 , un nouvel arrêté indiqua les vrais principes à adopter. La supériorité incontestable des races anglaises s'imposait à l'attention de tous les gens sérieux et l'Empereur fut un des premiers à en encourager l'introduction.

10 Le gouvernement de la Restauration continua à perfectionner les règles et un nouvel arrêté en 1820 précisa quelques points concernant les concurrents en fixant leur âge, leur taille et les poids qui devaient leur être attribués, ce qui commençait à homogénéiser les typologies des participants. De cette époque date la création d'un certain nombre de grands haras, notamment en région parisienne, tels que le haras de Meudon et le haras de Viroflay parmi les plus réputés.

11 Le Haras du Pin prit alors une importance considérable sous les ordres de son directeur Monsieur de Bonneval. Des missions, envoyées en Angleterre, ramenèrent en 10 ans une cinquantaine de reproducteurs pur-sang qui furent répartis sur l'ensemble du territoire.

12 En 1825, devant les succès obtenus par cette politique, il fallut à nouveau intervenir pour libérer et promouvoir quelque peu l'élevage français. Un décret de 1825 classait alors les chevaux de courses en deux catégories : ceux nés de père et de mère français et ceux nés de père et de mère étrangers. Ainsi fut créé le premier stud-book, ou registre d'origine, relatant toutes les naissances, en France, de chevaux de pur-sang.

13 À l'époque, on dénommait "pur-sang » les chevaux qui n'avaient que des ancêtres de sang pur dans les trois générations précédentes, aussi bien du côté du père que du côté de la mère. Malgré tout, la province continuait d'organiser des courses de chevaux, dit de pays, à l'image du Bidet en Bretagne ou du Roussin-voiturier-carrioleur en BasseNormandie, parmi d'autres.

Les efforts des gouvernements successifs pour guider l'amélioration de la production de chevaux de courses, exclusivement orientée vers le pur-sang dit anglais, et de plus en plus vers le pur-sang arabe, ne suffirent pas. Vers 1833, il y avait près de 1100 propriétaires de chevaux de courses en France qui faisaient courir sur les divers hippodromes du Royaume mais les résultats escomptés n'étaient pas au rendez-vous (fig. $\mathbf{n}^{\circ} \mathbf{1}$ ). 
Figure 1

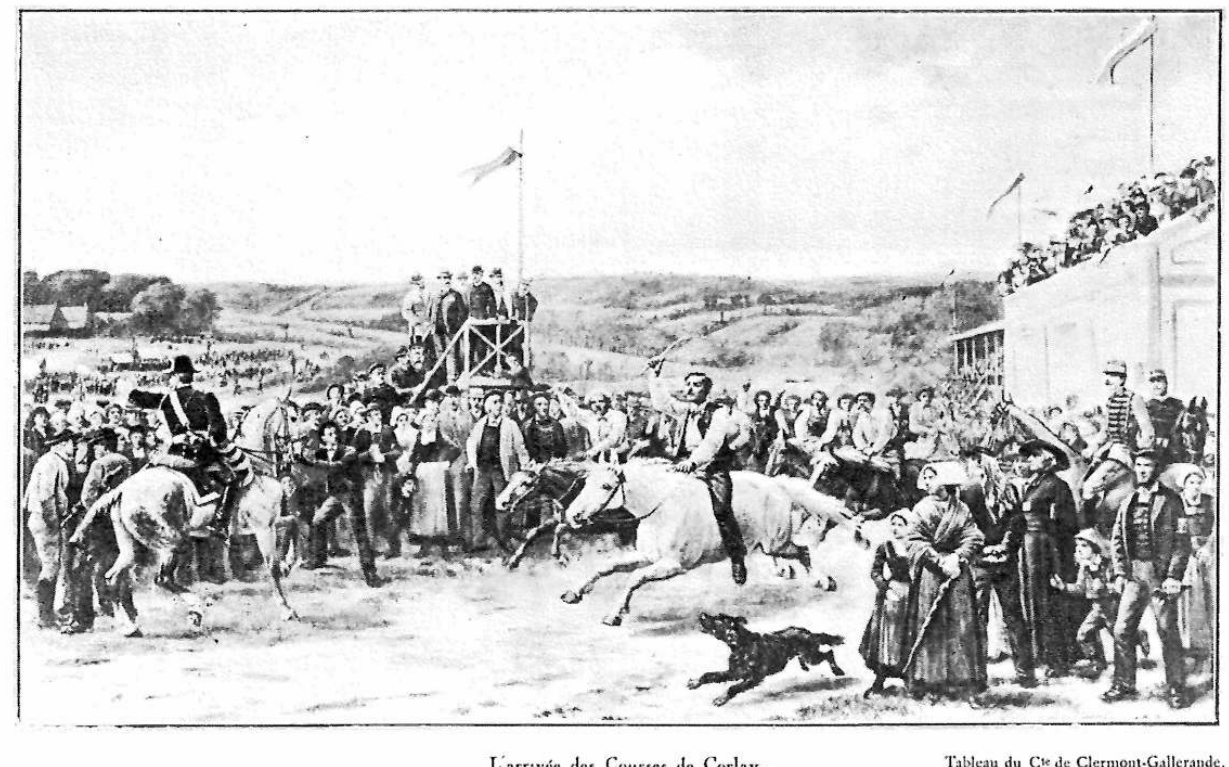

L'arrivée des Courses de Corlay. Tableau du Comte de Clermont-Gallerande. Carte postale ancienne. (c) Tanneguy de Sainte Marie.

C'est alors que les partisans du système anglais firent valoir qu'en France le gouvernement faisait tout, dirigeait tout, sacrifiait des sommes très importantes et n'obtenait rien; tandis qu'en Angleterre le gouvernement laissait libre cours aux initiatives des particuliers, ne dépensait que fort peu et n'en arrivait pas moins à des résultats admirables.

Ces constatations répétées et confirmées suscitèrent de nouvelles initiatives dont l'une des plus importantes fut la création de la «Société pour l'amélioration des races de chevaux en France » connue sous le nom de Jockey-Club. Cette nouvelle institution donna sa première journée de courses le 4 mai 1834 au Champ de Mars. Ce sera ensuite la résurrection de Chantilly avec le premier prix du Jockey-Club couru le 24 avril 1836 puis le premier prix de Diane créé en 1843.

17 Mais, Paris sera toujours Paris, et donc, ailleurs en France, il faudra se démarquer en inventant autre chose. Dans le haut du Cotentin M. Le Magnen, négociant en vins à Cherbourg, pense depuis longtemps que tous les anglo-saxons ne boivent pas que du thé. Il faut les attirer dans sa belle ville et les régates ne font plus recette. Or, le pays est peuplé de bidettes avec lesquelles on pourrait peut-être animer la ville. Le négociant se rapproche alors du maire de la ville, Noël Agnès, qui accepte d'allouer une subvention venant en complément d'une souscription pour le projet. Il sollicite également le directeur du haras national de Saint-Lô qui lui envoie son sous-directeur, un certain Ephrem Houël. Ce dernier réfléchissait déjà, depuis 1834, à une utilisation autre que le galop pour les chevaux normands qui, chaque semaine, se toisaient en ralliant le marché le plus proche dans un trot enlevé et allant.

Les 25 et 26 septembre 1836, se courront à Cherbourg sur la grève, plus précisément sur la plage de Collignon, de la gare maritime actuelle au port du Becquet, deux courses par 
jour, une au trot l'autre au galop. La distance était d'environ quatre kilomètres. Les départs étaient donnés en paquet et pour la course trottée, le galop étant interdit, les arrivées étaient plus spectaculaires car plus regroupées qu'avec les galopeurs. On voyait même quelques chevaux courir les deux spécialités. Les courses au trot étaient nées, remportant un franc succès à Cherbourg où elles seront reconduites chaque année. En 1837 on courra au trot à Caen où sera créée une autre société mère, La Société d'Encouragement à l'Élevage du Cheval Français, puis en 1838 à Saint-Lô.

Les pur-sang importés d'Angleterre avaient sailli nombre de juments autochtones et leurs produits, devenus Anglo-Normands, sont aujourd'hui à la base du stud-book trotteur français avec notamment le mythique Fuschia, né en 1833 à Saint-Côme-du-Mont dans la Manche. Père de près de 400 produits, il restera l'un des plus célèbres étalons du Haras du Pin de la fin de ce siècle (fig. ${ }^{\circ} \mathbf{2}$ ).

\section{Figure 2}
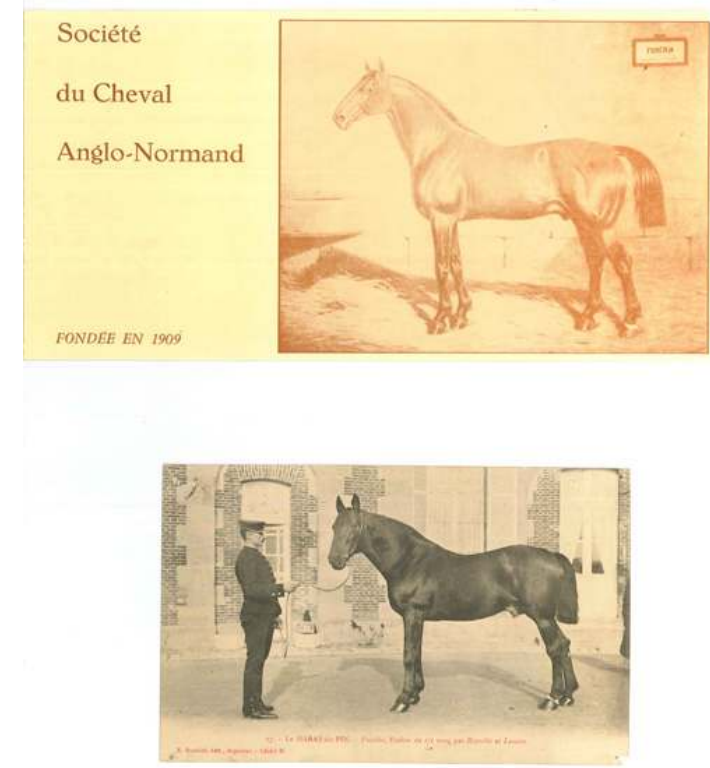

Société du Cheval Anglo-Normand fondée en 1909. En bas : Le Haras du Pin : Fuschia, étalon de demisang par Reynold et Lavater. Cartes postales anciennes.

(C) Tanneguy de Sainte Marie.

Promu directeur du dépôt d'étalons de Langonnet (Morbihan), Ephrem Houël rédige, en 1842, un traité complet de l'élève du cheval en Bretagne dans lequel il décrit l'ambiance des raids hippiques bretons (RED, en breton, veut dire course). Il encourage et mène, tambour battant, la création du champ de courses de Langonnet, aujourd'hui disparu (fig. n³). 


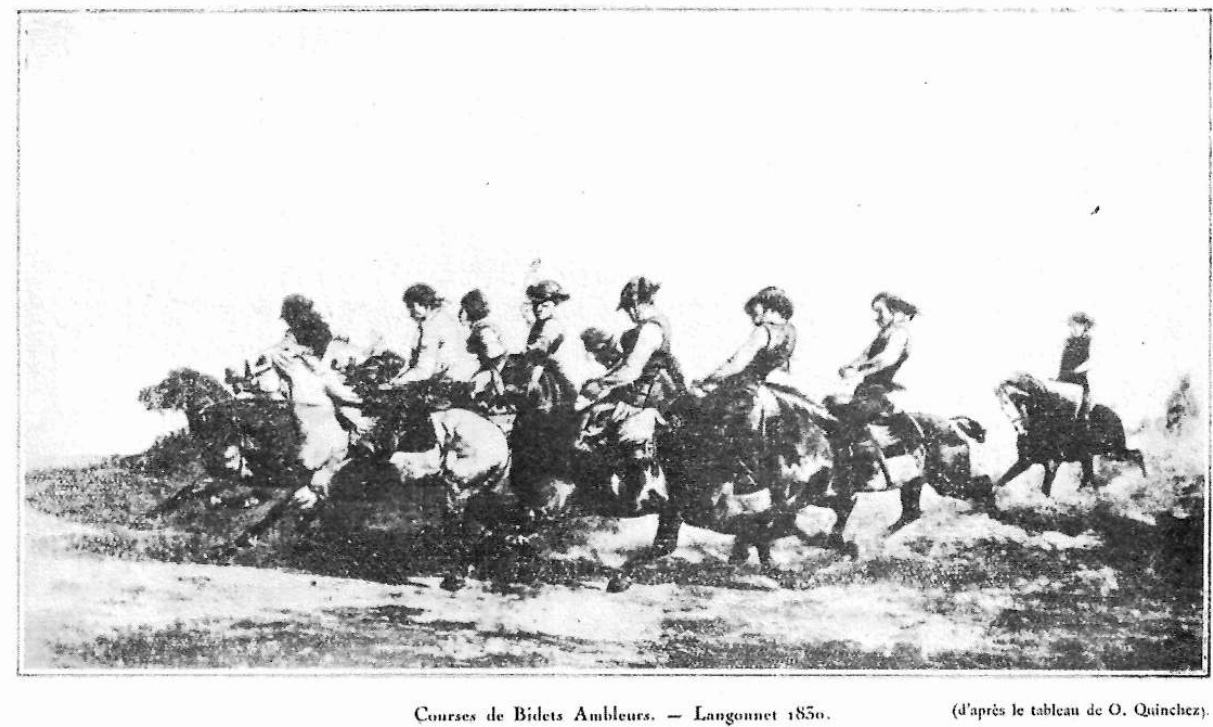

Courses de bidets Ambleurs, Langonnet 1830. Tableau d'O. Quinchez. Carte postale ancienne.

(c) Tanneguy de Sainte Marie.

21 Tout au long de ce XIX ${ }^{e}$ siècle, la forte implication des directeurs et sous-directeurs des haras dans la création et l'animation de champs de courses à proximité de leurs établissements fut remarquable sur tout le territoire (Cluny, Le Pin, Saint-Lô, Compiègne, Pompadour, Montier-en-Der, Angers, Blois, La Roche-sur-Yon, Lamballe, Pau, Tarbes...). Chacun aura son hippodrome et l'élevage local s'en développera d'autant.

À Langonnet, au sujet des courses de bidets, Ephrem Houel écrivait : « Rien n'est gracieux comme leur pose noble et assurée sur ces petits chevaux fringants et impatients qui se cabrent et mordillent leur frein rustique. Ils partent! souvent au nombre de dix à douze, quelquefois plus; mais quel terrain ont-ils à parcourir? C'est tantôt une route dure et inégale, tantôt des marais fangeux, tantôt des sentiers serpentant dans les rochers, là est un torrent à franchir, là une descente rapide suivie d'une montée aussi rude, rien ne les arrête, ils volent à travers les bruyères, les rochers, les ravins. Ils font ainsi une course de 4 à $5 \mathrm{~km}$. Le gagnant, héros de la fête, gagne deux moutons à Scaër, ou une génisse à Rostrenen, voire trois chèvres à Saint-Brieuc. On veut le nom du cheval, son âge, sa race, le nom de son père. On se croirait à New-Market, mais ce n'est pas pour $25000 \mathrm{~F}$ qu'il a couru, seulement pour un mouton à $3 \mathrm{~F}$. "

En 1847, le haras de Langonnet hébergeait dix-sept étalons pur-sang Anglais et un pursang arabe dans ses écuries.

On comptait en 1848 plus d'une cinquantaine de sociétés de courses de province où on courait au galop et au trot.

En 1856, la plaine de Longchamp, couvrant 66 hectares, remplacera le trop petit Champ de Mars et l'inauguration du nouvel hippodrome, flambant neuf, donnera lieu à une immense fête le 27 août 1857. À partir de 1861 le Duc de Morny invente Deauville dont l'hippodrome sera inauguré le 17 août 1864 . 
Le cheval de pur sang restait ainsi le roi de ces hippodromes mais les courses de province se développaient toujours et encore et les différents types de chevaux de courses se modelaient au fil des générations en fonction des utilisations qui en étaient faites.

Les relations extrêmement tendues qui rebondissaient d'année en année entre Maurice de Gasté, promoteur du seul et unique pur-sang Anglais à utiliser pour l'amélioration des races françaises et Olivier du Pontavice de Heussey, Directeur du Haras du Pin qui lui promouvait le demi-sang normand pour améliorer l'élevage du cheval de selle, coûteront le fauteuil de Directeur Général des Haras à ce dernier.

Notre directeur se plaisait à répéter que le pur-sang était un admirable, essentiel et inévitable améliorateur de la race anglo-normande, mais que, comme toute excellente chose, il pouvait y avoir, si on en abusait, de très graves inconvénients. Le pur-sang devait donc être utilisé avec discernement. Il devait ainsi être un parfum discret sans devenir un alcool fort (fig. $\left.\mathbf{n}^{\circ} \mathbf{4}\right)$.

Figure 4
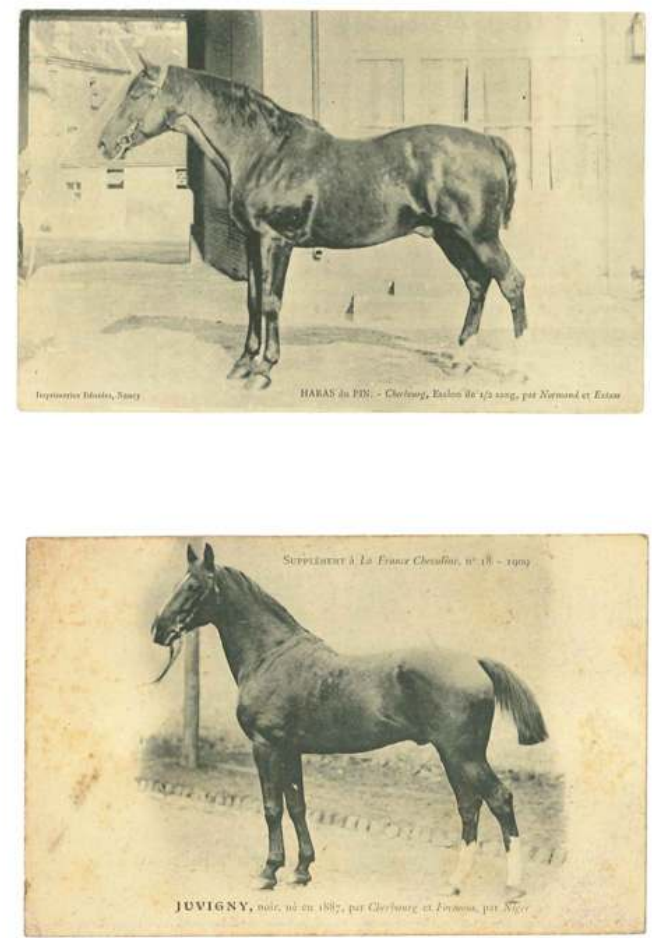

Haras du Pin : Cherbourg, étalon de demi-sang, par Normand et Extase. En bas : Juvigny, noir, né en 1887, par Cherbourg et Focmesa par Niger. Cartes postales anciennes.

(C) Tanneguy de Sainte Marie.

En cette seconde moitié du XIX ${ }^{e}$ siècle, les courses d'obstacles commençaient à avoir du succès après des débuts difficiles, ici ou là, dont les premiers steeple-chases courus sur le domaine du haras de La Croix de Berny (Hauts-de-Seine). Certaines provinces, s'étant spécialisées dans ce type de spectacle plus populaire, organiseront en 1862 quelque 122 steeples. Devant ces initiatives fort dynamiques mais quelque peu éparses, il fallut unifier les programmes et condenser les efforts. Ce sera l'œuvre de la Société des Steeplechases de France créée en 1863. 

l'importation d'étalons Norfolk croisés avec les demi-sang trotteurs, la recherche d'aliments et de fourrages de meilleure qualité, le développement des transports, notamment avec l'arrivée du chemin de fer à la fin du siècle, la mise en place de standards de races par l'intermédiaire de la tenue de stud-books et l'organisation toujours plus ordonnée des réunions de courses, se sont traduits par une modification très sensible des phénotypes des populations de chevaux concernées (fig. $\mathbf{n}^{\circ} 5$ ).

Figure 5
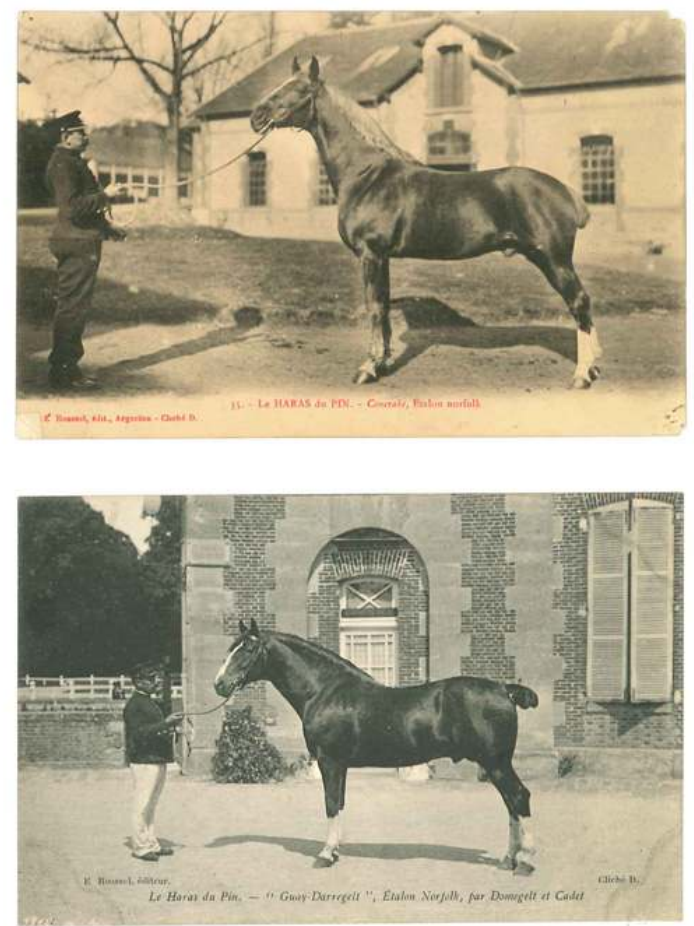

Haras du Pin : Concrate, étalon Norflok. En bas : Gay-Darregelt, étalon Norfolk, par Domegelt et Cadet. Cartes postales anciennes.

(c) Tanneguy de Sainte Marie.

Les tailles, les types, les cadres, les allures, les tempéraments ne sont plus les mêmes qu'il y a 150 ans. Le chanfrein busqué de l'Anglo-Normand, devenu Trotteur Français, a totalement disparu avec l'arrivée des étalons Trotteur-Américains. Le bidet barbu d'Armorique n'a plus besoin de se défendre contre l'ajonc car il est nourri avec du bon foin (fig. $\left.\mathbf{n}^{\circ} \mathbf{6}\right)$. 


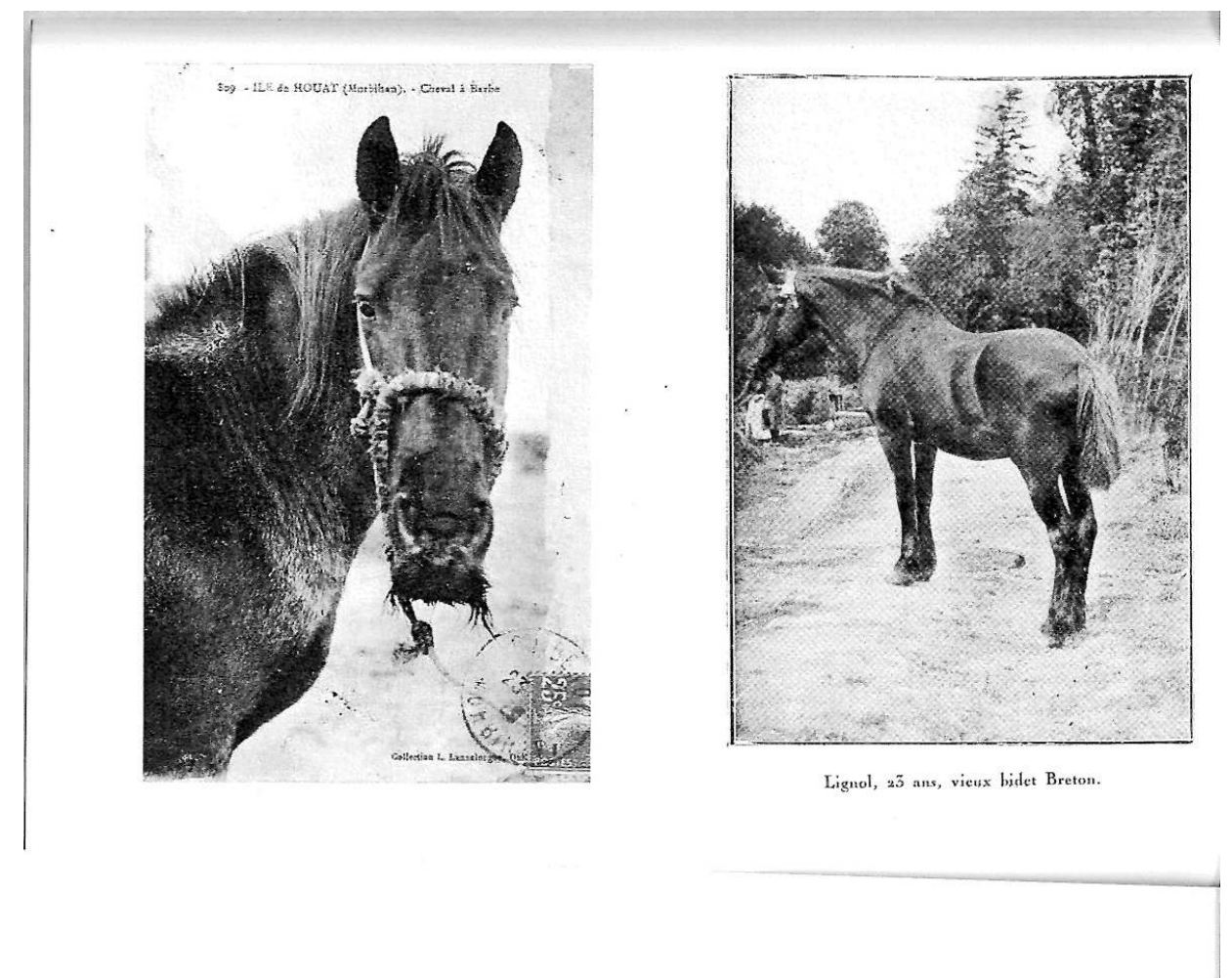

Île de Houat (Morbihan) : cheval à barbe. En bas : Lignol, 23 ans, vieux bidet Breton. Cartes postales anciennes.

(c) Tanneguy de Sainte Marie.

La loi de 1891, toujours en vigueur de nos jours, interdit, en son article 4, les paris sur les courses de chevaux faits avec tous venants en quelque lieu et sous quelque forme que ce soit. Dès l'article 5 , il est précisé qu'une exception à la règle édictée plus haut permet aux Sociétés de courses hippiques d'établir, avec autorisation et moyennant quelques prélèvements, l'organisation de paris mutuels sur leurs hippodromes.

Cette autorisation légale du pari mutuel, de cette «loterie officielle» comme on l'a appelée, devait amener avec fracas l'intervention des moralistes auxquels se joignirent, pour la circonstance, tous ceux qui condamnaient, dans son principe, l'institution même des courses de chevaux.

Pour la saison de monte de 1892, il est intéressant de lire attentivement les conditions d'inscription des juments aux douze principaux étalons trotteurs du haras du Pin. Ces étalons, parmi lesquels nous trouvons Fuschia (Grand-mère pur-sang), Cherbourg, Phaéton (fils du pur-sang anglais The Heir of Linne), Kachemyr (fils de Phaéton), Juvigny (fils de Cherbourg), Ilote (petit-fils de Phaéton), dont les origines ne font apparaître que les noms des pères et grands-pères maternels, sont réservés à quelques catégories de juments (fig. $\left.n^{\circ} 7\right)$. 
Figure 7
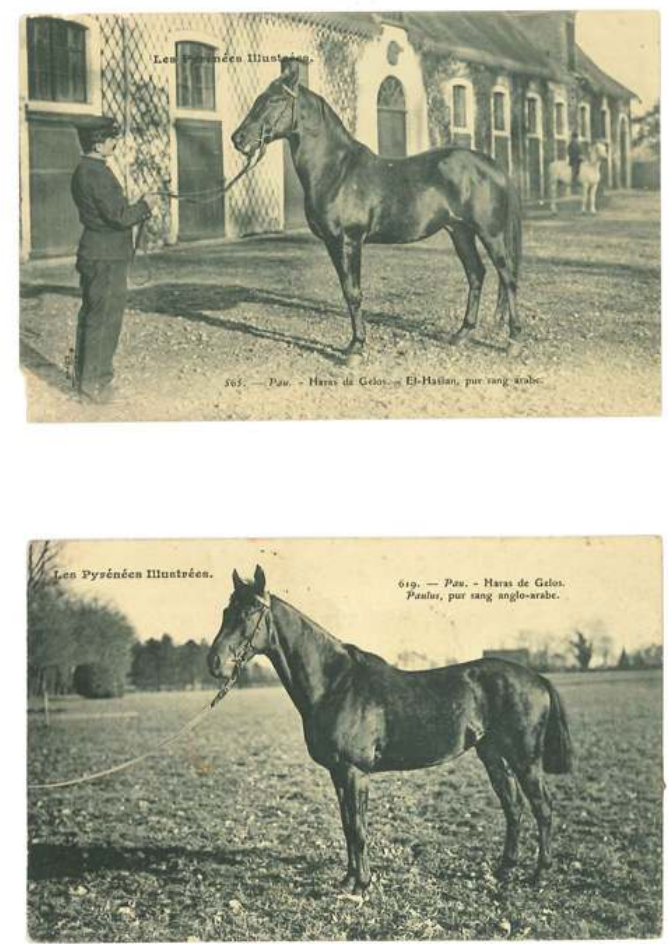

Pau, Haras de Gelos : El-Hassan, pur-sang arabe. En bas : Paulus, pur-sang anglo-arabe. Cartes postales anciennes.

(c) Tanneguy de Sainte Marie. dessus à 1'52" pour les 3 ans, on peut également faire bénéficier de ces saillies des juments de pur-sang ayant gagné au moins $3000 \mathrm{~F}$ en courses plates ou d'obstacles ou ayant produit des vainqueurs dans ces disciplines. Quant aux autres juments pouvant être servies, il n'est jamais fait référence à leur race mais seulement à leur palmarès et à ceux de leurs produits.

Les stud-books sont très largement ouverts ce qui est heureux pour l'amélioration de l'élevage. Tout a changé de nos jours et les sociétés mères sont vigilantes. Ne court plus qui veut, quand il veut, où il veut et ne croise pas qui veut avec ce qu'il veut (fig. $\mathbf{n}^{\circ} \mathbf{8}$ ). 

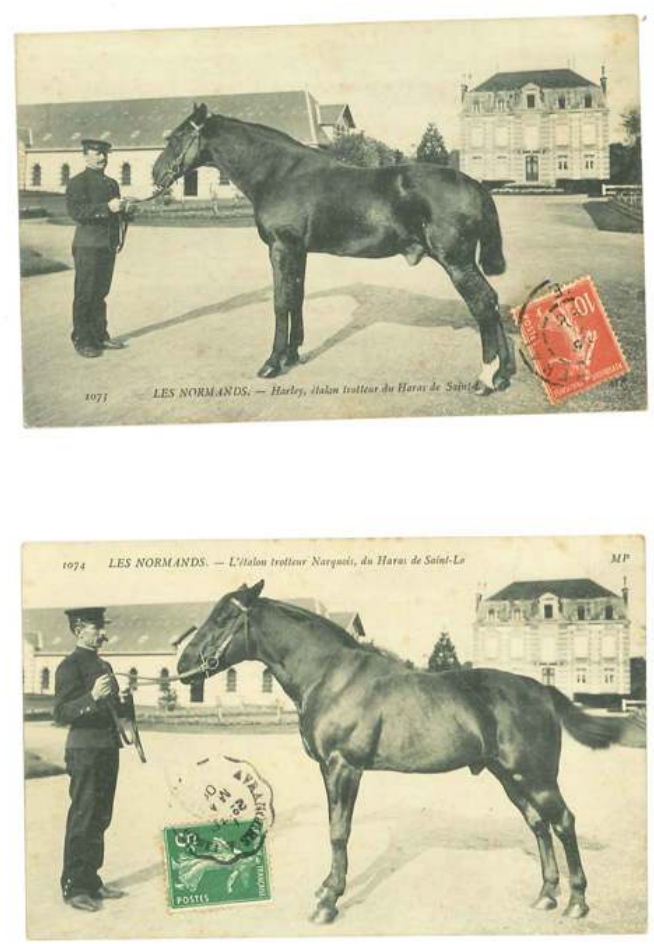

Les Normands : Harley, étalon trotteur du Haras de Saint-Lô. En bas : Narquois, étalon trotteur du Haras de Saint-Lô. Cartes postales anciennes.

(c) Tanneguy de Sainte Marie.

Et pourtant, en ce début du XXI ${ }^{\mathrm{e}}$ siècle, dans quelques contrées de France métropolitaine et d'Outre-mer, les courses locales perdurent ici et là. Sans parler des courses de Stockmen de Nouvelle-Calédonie, évoquons seulement la Bretagne occidentale, du Morbihan à Merlevenez, du Finistère à Bannalec et Clohars-Carnoet, des Côtes d'Armor à Plouguenast et quelques autres où les courses de chevaux, dits "du pays", héritières des raids hippiques, perdurent très activement sous la couverture d'arrêtés préfectoraux dérogatoires au code général des courses de chevaux.

En effet, deux importantes circulaires ministérielles encadrent et régissent les courses de chevaux en France :

- Celle du 31 mai 1946, qui interdit toute réunion de courses de chevaux non officielle.

- Celle du 24 juillet 1946, qui, sans démentir la précédente, laisse une certaine marge aux préfets dans le cadre de courses organisées avec des chevaux du pays et une seule fois par an. Étant entendu que cette tolérance n'est délivrée que sans pari mutuel ni jeu d'argent d'aucune sorte.

Citons pour exemple cette lettre du Préfet du Finistère, en date 5 août 2007, à un président d'une association bretonne organisant des courses de chevaux de pays :

«Monsieur le Président,

Vous avez sollicité l'autorisation d'organiser des courses hippiques sur la commune de Bannalec le 12 août prochain. Dans le cadre de la tolérance admise par le Ministère de l'Agriculture et de la Pêche, afin de ne pas nuire à l'animation des 
réjouissances populaires, je ne vois pas d'objections au déroulement de cette manifestation.

Cependant,

- Aucun pari mutuel, ni jeu d'argent d'aucune sorte ne sera organisé ;

- Ce type de manifestation doit conserver son caractère exceptionnel et ne peut avoir lieu qu'une fois l'an ;

- Les organisateurs ne devront poursuivre d'autre but que celui d'apporter un peu de distraction dans la localité ou un but charitable ou philanthropique à l'aide du produit des seules entrées qu'ils pourront percevoir ;

- Ces courses n'ont pas de caractère de courses publiques et le gagnant ne peut se voir attribuer un prix d'une valeur supérieure à 458 euros ;

- Les chevaux et les poneys devront être vaccinés contre la grippe équine ;

- L'organisateur assurera la sécurité des participants et du public.»

\section{RÉSUMÉS}

De tout temps, les courses de chevaux ont tiré et tirent encore vers le haut, paraît-il, la sélection et l'amélioration des meilleurs sujets. Au siècle des empires, des royaumes et des premières républiques, seuls le pur-sang arabe et le pur-sang anglais ne toléraient et ne tolèrent toujours pas de croisements dans leurs ascendants. Toutes les autres populations d'équidés de tous types ont subi les lois, les modes et les règlements de l'homme afin de s'adapter de gré et de force aux nouvelles utilisations, devenues "disciplines » que seuls les loisirs feront évoluer en ce XXI siècle. 


\section{AUTEUR}

\section{TANNEGUY DE SAINTE MARIE}

Ingénieur des haras nationaux, Haras du Pin tanneguy.de_sainte_marie@haras-nationaux.ifce.fr 